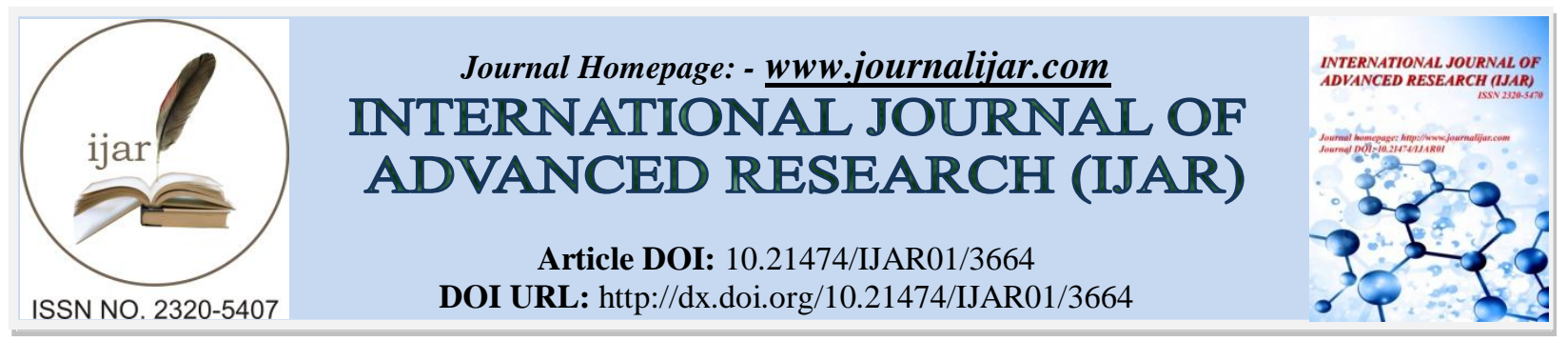

RESEARCH ARTICLE

\title{
CARBON NANOTUBES: SYNTHESIS, PROPERTIES AND TECHNOLOGICAL APLLICATIONS.
}

\author{
Demewez Amtate (Msc) ${ }^{1}$ and Girma Goro $(\mathrm{PhD})^{2}$. \\ 1. Department of Physics, Faculty of Natural and Computational Sciences, Assosa, University, P.o.box 18, \\ Assosa, Ethiopia. \\ 2. Department of Physics, Collage of Natural and Computational Sciences, Dire Dawa University.
}

\section{Manuscript Info}

Manuscript History

Received: 11 January 2017

Final Accepted: 01 February 2017

Published: March 2017

Key words:-

Carbon nanotubes, Chemical vapor deposition, Energy band structure.

\section{Abstract}

This project reviews synthesis, properties and technological applications of carbon nanotubes. The different synthesis methods of carbon nanotubes are illustrated briefly. The energy dependence of density of states was used to determine the optical properties of CNT. Finally, brief description of optical and electronic properties was presented. Using MATLAB Code the theoretical simulation of electronic band structure as a function of wave vector, the band gap as a function of tube diameter and the density of state as a function of energy band are generated.

Copy Right, IJAR, 2017,. All rights reserved.

\section{Introduction:-}

The history of nanotechnology is generally understood to have begun in December 1959 when physicist Richard Feynman gave a speech, "There are Plenty of Rooms at the Bottom" at an American Physical Society meeting at the California Institute of Technology in which he identified the potential of nanotechnology. Feynman said it should be possible to build machines small enough to manufacture objects with atomic precision, and that if information could be written on an atomic scale, "all of the information that man has carefully accumulated in all the books in the world can be written in a cube of material $12700 \mathrm{~nm}$ wide about the size of the smallest piece of dust visible to the human eye."

Nanotechnology and Nanoscience got a boost in the early 1980s with two major developments: the birth of cluster science and the invention of the scanning tunneling microscope (STM). This development led to the discovery of fullerenes in 1985 (Prasada et al., 2008) and carbon nanotube a few years later. In another development, the synthesis and properties of semiconductors was studied; this led to a fast increasing number of metal and metal oxide nanoparticles and quantum dots.

Nanotechnology as one of the most modern technologies has attracted many researchers all over the world to study the different aspects of nanosystems and nanostructures. Among various nanostructures, carbon nanotube (CNT) has an influential role in this technology due to its remarkable mechanical, electrical, thermal and chemical properties. This nanostructure has been employed in different fields including nanomaterial, nanoelectronics and nanomechanics during recent decades. Due to these extensive applications, its modeling and analysis in various conditions are more demanded (Fakhrabadi et al., 2012).

Carbon nanotubes (CNTs) are tubular structures that are typically of nanometer diameter and many micrometers in length. This fascinating new class of materials was first observed by Endo in 1975 and later by Iijima in 1991 in the

Corresponding Author:- Demewez Amtate.

Address:- Department of Physics, Faculty of Natural and Computational Sciences, Assosa, University, 
soot produced in the arc-discharge synthesis of fullerenes (Dresselhaus et al., 2004). The structure of a CNT can be described in terms of a single graphite layer (graphene) rolled up to form a single cylinder or concentrically arranged cylinders. The former is referred to as a single-wall nanotube (SWNT) and the latter are called multi-wall nanotubes (MWNTs). MWNTs can form starting from double-walled tubes (DWNTs) to tubes with 50 or more walls or layers.

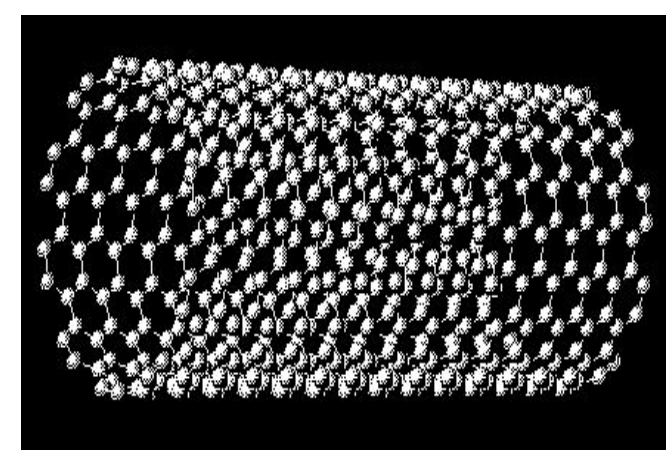

Single wall carbon nanotube

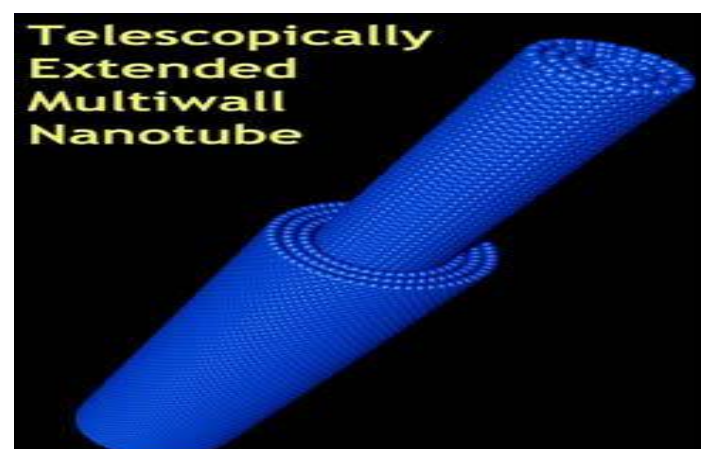

Multi wall carbon nanotube

Figure 1:- Structure of single-wall and multi-wall carbon nanotubes (Zhang et al., 2003).

Single-wall nanotubes has only one single layer and with a diameter of 1 to $5 \mathrm{~nm}$. The properties of SWNT are more stable than MWNT so it is more favorable. This is due to the degree of relative stability for carbon CNT bundle interconnects versus the length bundle and the diameter of each individual CNT has been obtained. The obtained results show that with increasing the length of the CNT bundle and the diameter of each individual CNT to be interconnected, then the relative stability increase, and the system become more stable (Fathi, 2009). MWNT is a little bigger than SWNT because MWNT has about 50 layers. MWNT's inner diameter is from 1.5 to $15 \mathrm{~nm}$ and the outer diameter is from $2.5 \mathrm{~nm}$ to $30 \mathrm{~nm}$. SWNT have better defined shapes of cylinder than MWNT, thus MWNT has more possibilities of structure defects and its nanostructure is less stable (Zhang et al., 2003).

It is a well-known fact that much of the Carbon Nanotubes produced are usually formed along with a mixture of amorphous carbon, fullerenes and other impurities. Cost effective and efficient ways of separation and filtration of Carbon Nanotubes are yet to be discovered. Amongst the available methodologies the three most commonly used techniques includes Arc-Discharge method, Laser Ablation method and Chemical Vapor Deposition (CVD). The CVD has proved to be a comparatively reliable way of synthesizing CNTs (Tripathi et al., 2010).

Within material science, the optical properties of carbon nano tubes refer specifically to the absorption, photoluminescence and Raman spectroscopy. Spectroscopic methods offer the possibility of quick and non-destructive characterization of relatively large amounts of carbon nanotubes. There is a strong demand for such characterization from the industrial point of view: since numerous parameters of the nanotube synthesis can be changed, intentionally or unintentionally, to alter the nanotube quality. Optical absorption, photoluminescence and Raman spectroscopes allow quick and reliable characterization of this nanotube quality in terms of non-tubular carbon content, structure (chirality) of the produced nanotubes, and structural defects. Those features determine nearly any other properties such as optical, mechanical, and electrical properties.

General Objective of this work being reported here was:-

$>$ To review the synthesis, properties and application of carbon nanotubes

\section{Specific Objectives were:-}

* To explain optical and electronic properties of carbon nanotubes

* To review the applications of carbon nanotubes

\section{Literature Review:-}

Fabrication of Carbon Nanotubes:-

Following the rapid advancement of carbon nanotubes and applications, different techniques to produce carbon nanotubes have been developed. Carbon nanotubes can be fabricated using techniques such as arc discharge, laser ablation, and chemical vapor deposition (CVD) methods. 


\section{Arc discharge method:-}

This was the first available method to fabricate carbon nanotubes. An electric arc discharge is an electrical breakdown of a gas producing a plasma discharge, very similar to a spark, which is the flow of current through a nonconductive medium such as air or an insulator. In a conventional carbon arc discharge fabrication, a plasma discharge is generated in a small gap between two graphite electrodes. In this method, carbon nanotubes are produced at the core in the cathode deposit (Javey et al., 2002).

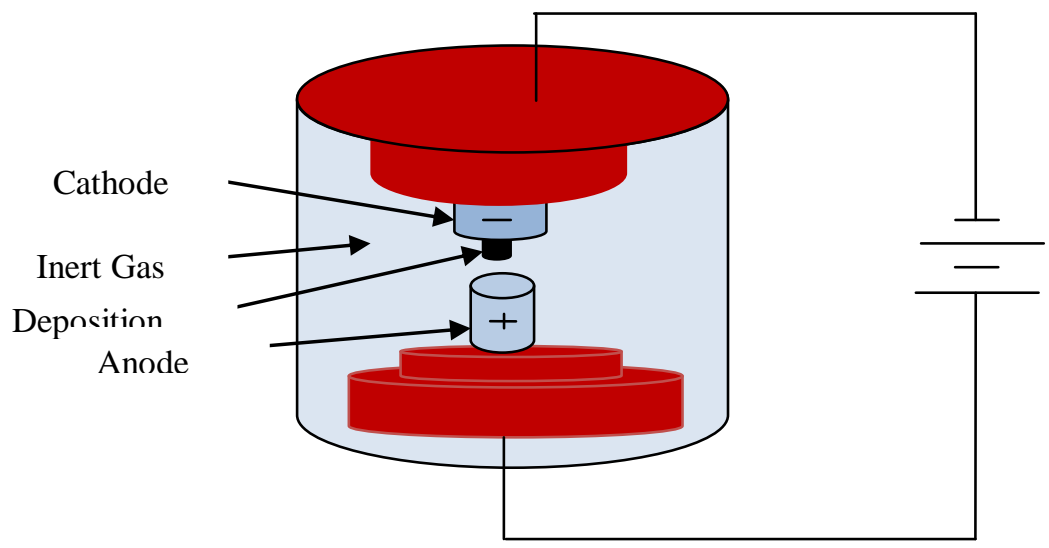

Figure 2:- Schematic diagram of an arc-discharge system

Source: http://students.chem.tue.nl/ifp03/synthesis.html.

As shown figure 2 during the arc discharge, two graphite rods are placed in an enclosure that is filled with some inert gas (like helium or argon) at low pressure (between 50 and $700 \mathrm{mbar}$ ). The carbon rods act as electrodes which are kept at different potentials. The anode is moved close to the cathode until an arc appears and the electrodes are kept at the distance of $1 \mathrm{~mm}$ for the whole duration of the process that takes about one minute. After the de-pressurization and cooling of the chamber, the nanotubes together with the by-products can be collected. Most nanotubes deposit on the cathode. The synthesis product yield which represents the amount of carbon nanotubes expected in the converted carbon is $60 \%$ (Isaacs et al., 2010).

\section{Laser ablation:-}

Laser ablation is a process of removing materials from a solid surface by irradiating the surface with a laser beam. It was developed in 1995 to produce CNTs. Figure 3 illustrates the growth setup of laser ablation. In a typical laser ablation process for CNT growth, the graphite block is placed inside an oven and hit by a pulsed laser, and Argon gas is pumped along the direction of the laser point. The oven temperature is set approximately to $1200^{\circ} \mathrm{C}$. As the laser ablates the target, carbon is vaporized and carried by the flowing gas onto a cool copper collector. CNTs grow on the cooler surfaces of the reactor as the vaporized carbon condenses. Sometimes, a water cooled surface may be included in the system to collect the CNTs. SWNTs are formed from a composite block of graphite and metal catalyst particles, whereas MWNTs form from pure graphite as the starting material (Guo et al., 1995).

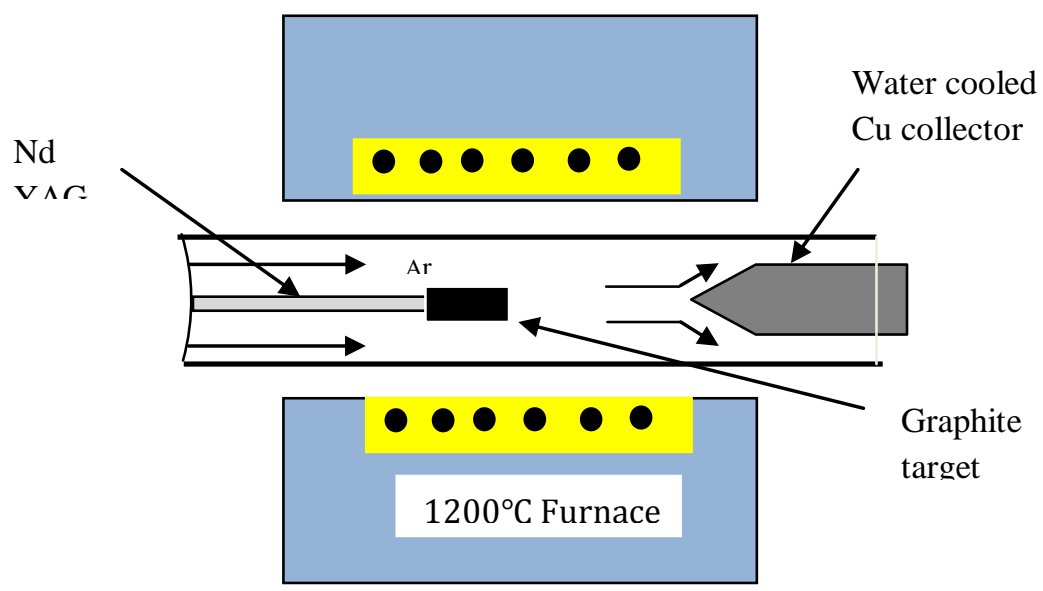

Figure 3:- Laser ablation equipment to produce CNTs. 
Source: http://students.chem.tue.nl/ifp03/synthesis.html

Disadvantages of Arc-Discharge and Laser ablation:-

Both arc-discharge and laser ablation produce some of the most high quality nanotubes but suffer from the following disadvantages, which limit their use as large scale industrial processes.

$\checkmark$ Both need extensive amount of energy to produce nanotubes. Such a huge amount of energy is not only impossible but also uneconomical for large scale production.

$\checkmark$ Both methods require solid carbon/graphite as a target which has to be evaporated to get nanotubes. It is difficult to get such large graphite to be used as target in industrial process which limits its exploitation as large scale process.

$\checkmark$ Both processes grow nanotubes in highly tangled form, mixed with unwanted form of carbon or catalysts. Thus CNTs produced by these processes require purification to get purified and assembled forms. The designing of such refining processes is difficult and expensive.

All the above mentioned factors severely limit the use of both arc-discharge and laser ablation as large scale processes for production of carbon nanotubes. In order to address these issues other methods were developed.

\section{Chemical vapor deposition (CVD) grown carbon nanotubes:-}

During CVD, a substrate covered with metal catalysts, like nickel, cobalt, iron, or a combination is heated to approximately $700^{\circ} \mathrm{C}$. As shown figure 4, the growth starts after two gases are passed through the chamber, a carrier gas like nitrogen, hydrogen or argon, and some hydrocarbon gas like acetylene $\left(\mathrm{C}_{2} \mathrm{H}_{2}\right)$ or methane $\left(\mathrm{CH}_{4}\right)$. The synthesis production yield, which indicates the amount of carbon nanotubes in the converted carbon, reaches $90 \%$ (Isaacs et al., 2010). CVD is commonly used for industrial purposes because the method is already well investigated and offers acceptable results on the industrial-scale.

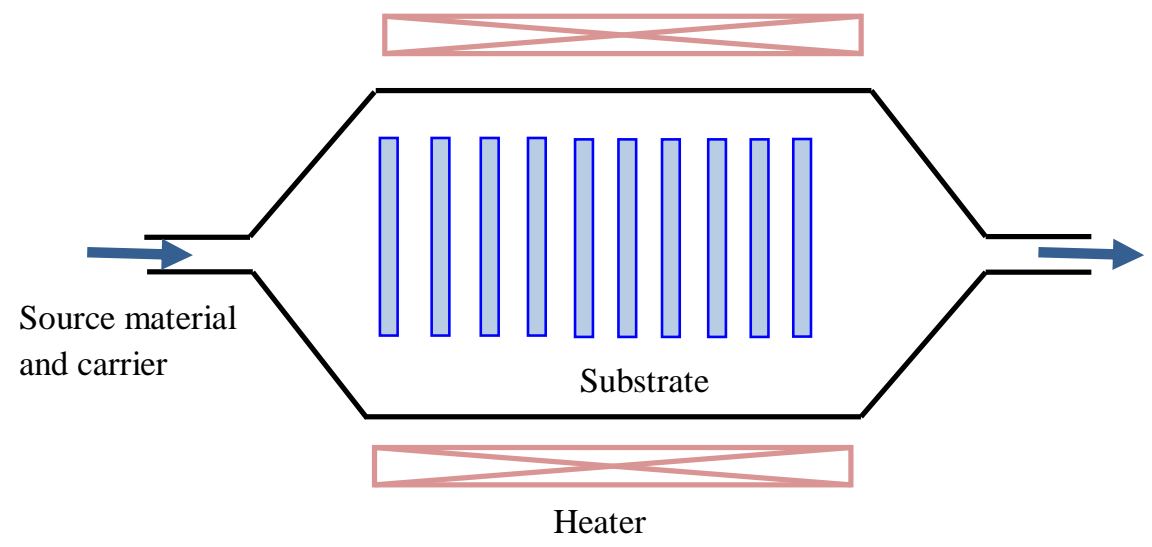

Figure 4:- Schematic diagram of chemical vapor deposition method.

Source: http://en.wikipedia.org/wiki/Chemical vapor deposition

\section{Advantages of Chemical vapor deposition:-}

This method has many advantages as compared to the arc discharge and the laser ablation method. To mention some:

$>$ Reaction process and reactor design is simple, reaction is easy to control and manipulate.

$>$ Raw materials are abundant and available readily in the form of gases.

$>$ The process does not needed huge amount of energy to produce high quality and large quantity of carbon nanotubes.

$>$ Process is capable of producing CNTs directly onto substrates which ease out the process of further collection and separation and eliminates post refining processes to a large extent. Some refining is required in some cases for further purification.

$>$ Process is unique for the production of vertically aligned nanotubes. No other process can produce aligned nanotubes. 
More and more research is underway in the world today for the production of large quantities of high purity carbon nanotubes by chemical vapor deposition process. Researchers are developing method and designing reactors, which could be utilized as units for large scale production of carbon nanotubes.

The major pitfall for CVD grown MWNTs is the high defect density in their structures. The defective nature of CVD-grown MWNTs remains to be thoroughly understood, but is most likely due to the relatively low growth temperature, which is not sufficient to provide adequate thermal energy to anneal nanotubes into perfectly crystalline structures.

\section{Properties of Carbon Nanotubes:-}

The wide range of electronic, optical, thermal, magnetic mechanical, chemical, physical and structural properties of carbon nanotubes vary according to the different diameter, chirality, length, and direction of 'twist' of the nanotube. Many applications arise from the surprising and desirable properties they exhibit, some of which are already being used in new and improved products. Among these properties of carbon nanotubes, optical and electronic properties are discussed in detail.

\section{Optical properties of carbon nanotubes:-}

The optical properties of CNTs are linked closely to their structural and electronic properties and are fully governed by one parameter, its chiral vector, which describes how the carbon-atom honeycomb is organized with respect to the CNT axis. Depending only on this parameter, CNTs may behave as a direct band gap semiconductor or as a metal.

Optical properties of carbon nanotubes derive from electronic transitions within one dimensional density of states (DOS). A typical feature of one-dimensional crystals is that their DOS is not a continuous function of energy, but it descends gradually and then increases in a discontinuous spike. The sharp peaks found in one-dimensional materials are called van Hove singularities. These van Hove singularities would be discussed later in the discussion part.

For an individual CNT, the optical properties of single walled (SWNTs and MWNTs should be considered separately. The optical properties of SWCNTs, especially those with diameters less than $1 \mathrm{~nm}$, exhibit very strong dependence on the detailed atomic structure (chirality) (Bachilo et al., 2002). Chirality-controlled CNT growth or chirality separation is still difficult in experiments. Thus SWCNT arrays usually contain much different chirality with a random distribution, which complicates the engineering of optical properties and functionalities. In contrast, MWCNTs, due to their larger size, have more regular and uniform optical properties. It is thus reasonable to treat each MWCNT as a homogeneous medium and design the structure for different applications (Lidorikis and Ferrari, 2009).

The optical properties of a CNT array further depend on the manner in which the CNTs are arranged, including pattern and intertube distance. Vertical arrays of nanotubes and nanowires have attracted much attention recently in solar, photonic crystal, mode-locked fiber lasers, and nanoantenna applications. For example, absorption enhancement in silicon nanowire arrays has been predicted using electromagnetic calculations and observed in experiments. Also, anisotropic optical scattering is predicted and observed for an individual semiconductor nanowire. In comparison, MWNT arrays possess a similar cylinder array structure. Although enhanced absorption and anisotropic scattering are experimentally observed, they have been less investigated theoretically, and quantitative analysis is still lacking (Ruan et al., 2010).

\section{Raman spectroscopy:-}

Raman spectroscopy is one of the most powerful tools for characterization of carbon nanotubes. Without sample preparation, a fast and non-destructive analysis is possible. All allotropic forms of carbon are active in Raman spectroscopy (Arepalli et al., 2004): fullerenes, carbon nanotubes, amorphous carbon and polycrystalline carbon.

Raman spectroscopy provides information about molecular vibrations that can be used for sample identification and quantification. The technique involves shining a monochromatic light source that is laser on a sample and detecting the scattered light. The majority of the scattered light is of the same frequency as the excitation source; this is known as Rayleigh or elastic scattering. A very small amount of the scattered light is shifted in energy from the laser frequency due to interactions between the incident electromagnetic waves and the vibration energy levels of the molecules in the sample. 
Raman scattering offers a unique tool to characterize carbon materials such as graphite, diamond and carbon nanotubes, since the amount of ordering and degree of $s p^{2}$ and $s p^{3}$ bonding leaves a unique Raman fingerprint. Therefore, Raman spectroscopy is used here to identify carbon nanotubes and to characterize the obtained carbon structures by their degree of crystallinity.

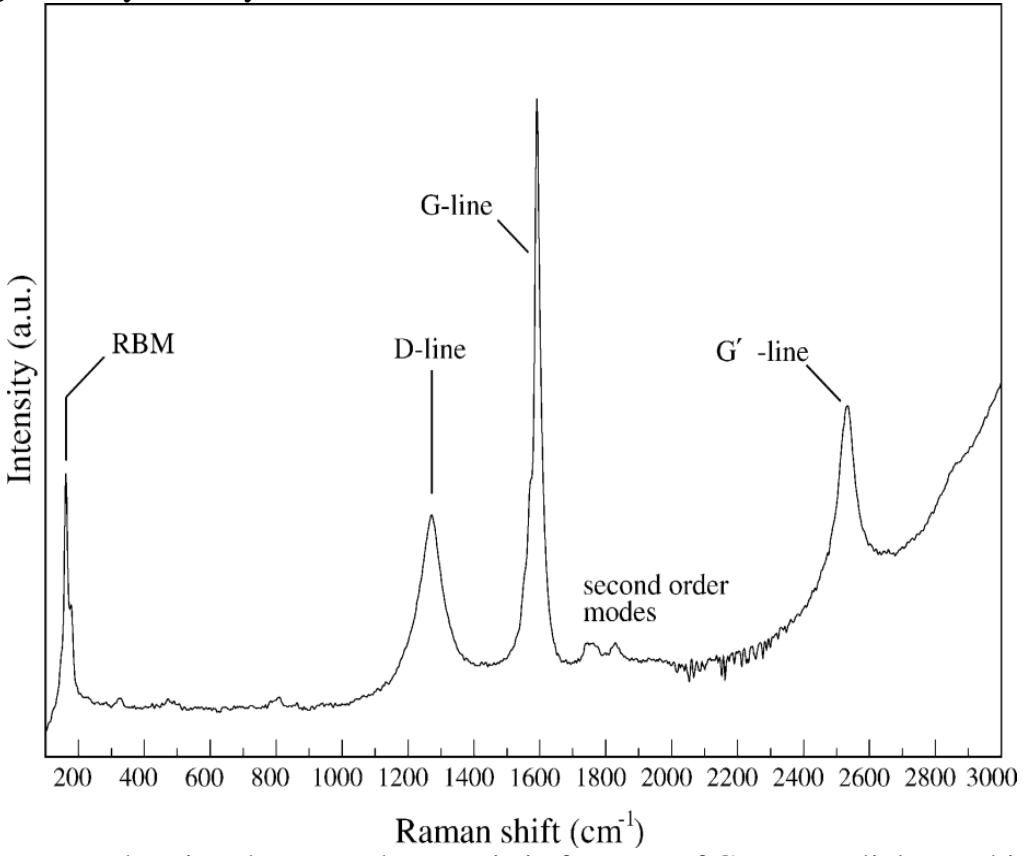

Figure 5:- Raman spectrum showing the most characteristic features of CNTs: Radial Breathing Mode (RBM), the D band, G' band and G band (Belin and Epron, 2005)

From figure 5 Raman spectrums showing the most characteristic features of CNTs are summarized as follows:

$>$ A low-frequency peak $<200 \mathrm{~cm}^{-1}$ (or bunch of peaks for poly disperse samples) when resonating conditions were met characteristic of the SWNT assigned to an $\mathrm{A}_{1 \mathrm{~g}}$ breathing mode of the tubes, whose frequency depends essentially on the diameter of the tube (RBM: radial breathing mode).

$>$ A large structure $\left(1340 \mathrm{~cm}^{-1}\right)$ assigned to residual ill-organized graphite, the so-called D-line (D: disorder).

$>$ The D- line or D-band is observed in the Raman spectra of semiconducting and metallic SWNT at the single nanotube level. The D-band in graphite involves scattering from a defect which breaks the basic symmetry of the graphene sheet, it is observed in $\mathrm{sp}^{2}$ carbons containing porous, impurities or other symmetry-breaking defects.

$>$ A high-frequency bunch (between 1500 and $1600 \mathrm{~cm}^{-1}$ ) called $\mathrm{G}$ band also characteristic of nanotubes, corresponding to a splitting of the $\mathrm{E}_{2 \mathrm{~g}}$ stretching mode of graphite.

$>$ A second order observed mode between 2450 and $2650 \mathrm{~cm}^{-1}$ assigned to the first overtone of the D mode and often called G mode; a combination mode of the D and $\mathrm{G}$ modes between 2775 and $2950 \mathrm{~cm}^{-1}$.

Radial breathing mode (RBM) corresponds to radial expansion-contraction of the nanotube. Therefore, its frequency $\boldsymbol{v}_{\mathrm{RBM}}$ (in $\mathrm{cm}^{-1}$ depends on the nanotube diameter $d$ (in $\mathrm{nm}$ ) and can be estimated (Fantini et al., 2004).

$v_{R B M}=\frac{234}{d}+10$ for SWNT or $v_{R B M}=\frac{234}{d}$ for DWNT, which is very useful in deducing the CNT diameter from the RBM position.

Raman spectroscopy for the RBM is therefore often used to determine the diameter or diameter distribution in SWNT samples and is further used to assign the chiral index $(n, m)$ of individual SWNTs by considering their resonant transitions.

\section{Nonlinear optical properties:-}

Non-linear optics (NLO) is the branch of optics that describes the behavior of light in non-linear media, that is media in which the dielectric polarization $\mathbf{P}$ responds nonlinearly to the electric field $\mathbf{E}$ of the light. This nonlinearity is typically only observed at very high light intensities (values of the electric field comparable to interatomic electric fields, typically $10^{8} \mathrm{~V} / \mathrm{m}$ ) such as those provided by pulsed lasers. 
The rapid development of nanoscience and nanotechnology provides lots of new opportunities for nonlinear optics. A growing number of nanomaterials have been shown to possess remarkable nonlinear optical (NLO) properties, which promotes the design and fabrication of nano and nanoscale optoelectronic and photonic devices (Hasan et al., 2009; Loh et al., 2010). The wonderful carbon allotropes discovered in recent decades are the most representative products of nanotechnology: from 3D carbon nanoparticles (graphite), to OD fullerenes, to 1D carbon nanotubes (CNTs), and then to 2D graphenes discovered most recently. Interestingly, all of these nano-carbons exhibit diverse NLO properties. For instance, carbon black suspensions show strong thermally-induced nonlinear scattering (NLS) effect and hence optical limiting (OL). For intense ns laser pulses fullerenes show large third-order optical nonlinearity and reverse saturable absorption (RSA) at certain wavelength band. CNTs show ultrafast second- and third order nonlinearities and saturable absorption (SA) in the near infrared (NIR) region and graphenes show ultrafast carrier relaxation time and ultra-broad-band resonate NLO response.

Materials with large second or third order optical nonlinearities are of great interest for data processing in photonic computing. Since the invention of laser in 1960 by Maiman and the first discovery of nonlinear optical (NLO) effect of quartz crystals, there has been a great deal of research directed at finding new materials with higher order NLO properties. Only noncentrosymmetric or polar materials could possess second order NLO susceptibility. Most third order NLO materials are generally too low for practical applications. Since the discovery of carbon nanotubes, which possess optimal properties (like highest thermal conductivity, Young modulus 100 times higher than steel, zero bandgap metal, ballistic conductance, simple structure, nanometer-size and ultra-light weight) by a NEC scientist Sumio Iijima in 1991, a heat of studying and engineering the properties of carbon nanotubes has been raised worldwide.

\section{Electronic properties of carbon nanotubes:-}

Carbon nanotubes (CNTs) provide a number of unique and special properties that suggest great promise for nanoelectronics applications. In particular, the high electrical conductivity of quantum wires provides a potential solution for on chip interconnect metals and transistors of future integrated circuits.

One crucial obstacle to overcome in fabrication is controlling whether the CNT is metallic or semiconducting. The critical parameter determining the electronic properties of CNTs is the chiral vector, $c_{h}=n a_{1}+m a_{2} \equiv(n, m)$, where $n$ and $m$ are integers and $a_{1}$ and $a_{2}$ are the real space unit vectors of the graphene sheet. When $n-m$ a multiple of 3, the simple theory leads to a crossing of bands at the Fermi energy, implying that CNT is metallic; otherwise, it is expected to be a semiconductor. Thus, armchair $(n, n)$, CNTs are expected to always be metallic, whereas zigzag $(n, 0)$, CNTs are semiconductor except when $\mathrm{n}$ is a multiple of 3 .

Since a carbon nanotube (CNT) is a rolled grapheme sheet, the band structure of CNT is also predetermined by electronic structure of graphene. However in contrast to graphene, CNT is a quasi-one-dimensional structure, in which electronic properties depends on how the graphene sheet was rolled up. To classify the variety of ways to wrap the nanotube from a graphene sheet two indexes, $n$ and $m$ are commonly used (Saito et al., 2000). Figure 6 illustrates an example of graphene sheet about to be wrapped into a nanotube with the primitive translation vectors $a_{1}$ and $a_{2}$. once wrapped, the length of the chiral vector becomes the tube circumference and the tube diameter $d$ can be calculated from this circumference as:

$d=\frac{a}{n} \sqrt{n^{2}+n m+m^{2}}$

The chiral angle is the angle between the translation vector $c_{h}$ and the $(n, 0)$ line as seen in figure 7 :

$\alpha=\arctan \left(\frac{\sqrt{3} n}{2 m+n}\right)$

Defined by the integer index pair, (n,m), the chiral angle ranges from 0 to $30^{\circ}$. Zigzag nanotubes have chiral angles of 0 degrees, armchair nanotubes have chiral angles of $30^{\circ}$ and chiral nanotubes have chiral angles between $0<$ $\alpha<30^{\circ}$. 


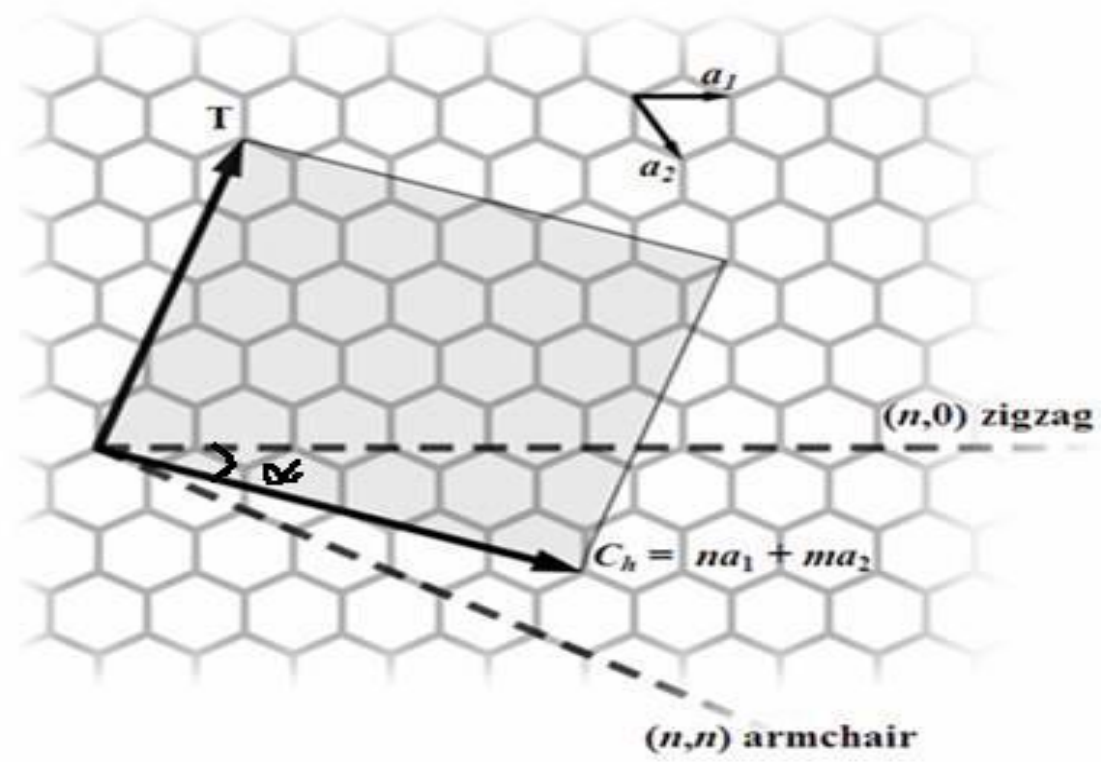

Figure 6:- Graphene model of a CNT showing the index pair notation used to characterize the CNT geometry. Source: http://en.wikipedia.org/wiki/ Carbonnanotube

$a=2.46 \AA$ from the graphene structure and by convention $n \geq m$. If $m=0$, the nanotubes are called zigzag, while if $n=m$, the nanotubes are called armchair. Otherwise, they are called chiral. Therefore varying the way of the rolling up one can arrive at CNT of different chirality and hence to different periodicity of the electronic wavefunction. In the electronic spectrum, such a periodicity manifests itself as delta-shaped peaks known as van Hove singularities which positions depends on a diameter and chirality of the SWNT. In particular the diameter is responsible for the band gap or type (metal or semiconducting) of the nanotube (Kataura et al., 1999). The electronic bands with the corresponding density of one-electron states (DOS) calculated for metallic and semiconducting single walled carbon nanotubes.

The electronic and optical properties of the tubes are considerably influenced by the environment (Charlier et al., 2001). Under externally applied pressure, the small interaction between the tube walls results in the internal tubes experiencing reduced pressure (Puech et al., 2004). The electronic transition energies are in the infrared and visible spectral range.

Generally, one can view carbon nanotubes as giant conjugated molecular wires with conjugation length corresponding to the whole length of the tube. In order to understand their electronic structure, we have to start with graphene, a single sheet of graphite. Carbon has four valence electrons of which three are strongly bound to neighbor atoms giving graphene its very high in-plane rigidity. The fourth electron is delocalized and shared by all the atoms, thus allowing for electronic current transport. However, because of its particular structure, graphene is electronically between semiconductor and metal. It is a semimetal or a zero-gap semiconductor.

\section{Application of Carbon Nanotubes:-}

A carbon nanotube is inert, has a high aspect ratio and a high tensile strength, low mass density, high heat conductivity, a large surface area, and a versatile electronic behavior, including high electron conductivity. However, while these are the main characteristics of individual nanotubes, many of them can form secondary structures such as ropes, fibers, papers and thin films with aligned tubes, all with their own specific properties. These properties make them ideal candidates for a large number of applications provided their cost is sufficiently low. The cost of carbon nanotubes depends strongly on both the quality and the production process. High-quality single-wall carbon nanotubes can cost 50-100 times more than gold. However, carbon nanotube synthesis is constantly improving, and sale prices are falling rapidly. The application of carbon nanotubes is therefore a very fast moving field, with new potential applications found every year, even several times per year. 


\section{Photovoltaic solar cells:-}

Carbon nanotubes (CNTs) have been widely used in solar cells research, for example, CNTs have been integrated in organic photovoltaic devices both as an electron acceptor material and as a transparent electrode. This is because the high conductivity along the tube axis of CNTs helps carrier's separation and collection. In 2005, GE Global Research observed a photovoltaic effect in a pristine nanotube diode device consisting of two CNTs with different electrical properties (Lee, 2005).

Photovoltaic device is a device that converts the energy of light directly into electricity by the photovoltaic effect. It is a crucial part of solar cells. Currently, wafer-based silicon (single crystal, poly crystalline and multicrystalline) solar cells and thin film solar cells based on amorphous silicon, CdTe, CuInGaSe ${ }_{2}$, and III-V semiconductors dominate photovoltaic manufacturing (Singh et al., 2008; Gupta et al., 2009). However, they are of low efficiency and expensive, which have limitations for replacement of current energy sources. There is a clear need to look for low-cost and high-efficiency solar cells. Many new kinds of solar cells have been proposed, such as p-n junction solar cells, dye-sensitized solar cells and organic solar cells.

Nanomaterials have been widely used in the above proposed solar cells. The advantages of using nanostructurebased solar cells are, on one hand, their high manufacturing costs as a result of using a low temperature process similar to printing instead of the high temperature vacuum deposition process typically used to produce conventional cells made with crystalline semiconductor material, and on the other hand, their low quantum efficiency which can be improved by using multiple electron-hole pair generation in nanostructures, like quantum dots and carbon nanotube ( Hanna et al., 2004; Jin Kim et al., 2008; Stubbs1 et al., 2010).

Recently, carbon nanotube (CNT) and graphene are playing an important role in such nanostructure-based solar cells. The dispersion of CNTs in a solution of an electron donating conjugated polymer is perhaps the most common strategy to implement CNT materials into organic photovoltaic devices to obtain higher efficiency. Enhancements of more than two orders of magnitude have been observed in the photocurrent from adding the single walled carbon nanotube to the poly matrix (Kymakis et al., 2003). The use of CNTs in DSSC has doubled the efficiency of this kind of photoelectrochemical solar cells (Kongkanand et al., 2007).

Graphene thin films also have been used as anode in dye-sensitized solar cells and organic solar cells layered graphene for photovoltaic devices have been reported to have an even better photovoltaic performance than the single walled carbon nanotube/quantum dots devices (Guo et al., 2010).

As one of major variables of DSSC, the dye absorbed light energy transits from ground state to excited state and gets electron injection. Electrons are injected in a very fast speed of femtosecond or picoseconds unit and oxidized dye is renewed within several nanoseconds. On the other hand, since rejoining speed that the electron becomes dissolved into electrolyte via surface state is slow such as micro seconds or milliseconds and most of photons are injected semiconductor conduction band, those electrons that are not injected meet holes again to be restored and decrease efficiency of solar cell. If we utilize CNT that is advantageous for electrical and thermal conduction as a compound material, we can increase electron injection speed than rejoining speed and increase efficiency of dye sensitized solar cells through movement of more electrons.

Since multi walled carbon nanotube (MWNT) is a material to transfer electricity and heat well, it can function as a basic electrode. Nanotubes function as electrodes to penetrate into broad $\mathrm{TiO}_{2}$ surface mutually and assist extracting charge carriers efficiently from dye layer. Since these electrodes are very clear under longer wavelength, they are advantageous for solar light spectrums.

\section{Chemical sensors:-}

The electrical conductance of semiconductor SWNTs was recently demonstrated to be highly sensitive to changes in the chemical composition of the surrounding atmosphere at room temperature, due to charge transfer between the nanotubes and the molecules from the gases adsorbed onto SWNT surfaces. It has also been shown that there is a linear dependence between the concentration of the adsorbed gas and the change in electrical properties, and that the adsorption is reversible. SWNT-based chemical $\mathrm{NO}_{2}$ and $\mathrm{NH}_{3}$ sensors are characterized by extremely short response times unlike conventional sensors (Kong et al., 2000; Chang et al., 2001). 
The electrical response has been measured by exposing MWNT films to sub-ppm $\mathrm{NO}_{2}$ concentrations (10-100 ppb in dry air) at different operating temperatures ranging between 25 and $215^{\circ} \mathrm{C}$ (Cantalini et al., 2003). For SWNTs, the sensor responses are linear for similar concentrations, with detection limits of $44 \mathrm{ppb}$ for $\mathrm{NO}_{2}$ and $262 \mathrm{ppb}$ for nitro toluene ( $\mathrm{Li}$ et al., 2003). High sensitivity to water or ammonia vapor has been demonstrated on a $\mathrm{SWNT}_{-} \mathrm{SiO}{ }_{2}$ composite (Varghese et al., 2001). This study indicated the presence of p-type SWNTs dispersed among the predominantly metallic SWNTs, and that the chemisorptions of gases on the surface of the semiconductor SWNTs is responsible for the sensing action.

Generally speaking, the sensitivities of these new nanotube-based sensors are three orders of magnitude higher than those of standard solid state devices. Another reason for using nanotubes instead of current sensors is their simplicity, the facts that they can be placed in very small systems and that they can operate at room temperature, as well as their selectivity. These advantages allow a limited number of sensor device architectures to be built for a variety of industrial purposes, while the current technology requires a large variety of devices based on mixed metal oxides, optomechanics, catalytic beads, electrochemistry, and so on.

\section{Multifunctional Materials:-}

One of the major benefits expected from incorporating carbon nanotubes into other solid or liquid materials is that they endow the material with some electrical conductivity while leaving other properties or behaviors unaffected. As already mentioned in the previous section, the percolation threshold is reached at very low nanotube loadings. Tailoring the electrical conductivity of a bulk material is then achieved by adjusting the nanotube volume fraction in the formerly insulating material while making sure that this fraction is not too large. As demonstrated by (Maruyama and Alam, 2002), there are three areas of interest regarding the electrical conductivity:

* Electrostatic discharge (for example, preventing fire or explosion hazards in combustible environments or perturbations in electronics, which requires an electrical resistivity of less than $1012 \Omega \mathrm{cm}$ ).

* Electrostatic painting (which requires the material to be painted to have enough electrical conductivity - an electrical resistivity below $106 \Omega \mathrm{cm}$ to prevent the charged paint droplets from being repelled).

* Electromagnetic interference shielding (which is achieved for an electrical resistivity of less than $10 \Omega \mathrm{cm}$ ).

Materials are often required to be multifunctional; for example, to have both high electrical conductivity and high toughness, or high thermal conductivity and high thermal stability. An association of several materials, each of them bringing one of the desired features, generally meets this need. The exceptional features and properties of carbon nanotubes make them likely to be a perfect multifunctional material in many cases. For instance, materials used in satellites are often required to be electrical conductive, mechanically self-supporting, able to transport away excess heat, and often to be robust against electromagnetic interference, while being of minimal weight and volume. All of these properties should be possible with a single nanotube containing composite material instead of complex multilaterals combining layers of polymers, aluminum, copper, and others.

The special nature of carbon combines with the molecular perfection of single-wall CNTs to endow them with exceptional material properties, such as very high electrical and thermal conductivity, strength, stiffness, and toughness. No other element in the periodic table bonds to itself in an extended network with the strength of the carbon-carbon bond. The delocalized pi-electron donated by each atom is free to move about the entire structure, rather than remain with its donor atom, giving rise to the first known molecule with metallic-type electrical conductivity. Furthermore, the high-frequency carbon-carbon bond vibrations provide an intrinsic thermal conductivity higher than even diamond.

In most materials, however, the actual observed material properties - strength, electrical conductivity, etc. - are degraded very substantially by the occurrence of defects in their structure. For example, high-strength steel typically fails at only about $1 \%$ of its theoretical breaking strength. CNTs, however, achieve values very close to their theoretical limits because of their molecular perfection of structure. This aspect is part of the unique story of CNTs. CNTs are an example of true nanotechnology: they are only about a nanometer in diameter, but are molecules that can be manipulated chemically and physically in very useful ways. They open an incredible range of applications in materials science, electronics, chemical processing, energy management, and many other fields.

CNTs Ceramic Applications, CNTs Air and Water Filtration, CNTs Biomedical Applications, CNT Catalyst Supports, CNTs Fibers and Fabrics, CNTs Structural Composites, CNTs Thermal Materials, CNTs Molecular Electronics, CNTs Conductive Adhesives and Connectors, CNTs Energy Storage, CNTs Conductive Plastics, CNTs 
Field Emission Applications, and CNTs Thermal Conductivity are the application of this nanotube which is responsible for the current manufacturing industry, agricultural industry and also many other industry.

\section{Other CNT Applications:-}

There is a wealth of other potential applications for CNTs, such as solar collection; nanoporous filters; catalyst supports; and coatings of all sorts. There are almost certainly many unanticipated applications for this remarkable material that will come to light in the years ahead, and which may prove to be the most important and valuable ones of all. Many researchers are looking into conductive and or water proof paper made with CNTs. CNTs have also been shown to absorb Infrared light and may have applications in the I/R Optics Industry.

\section{Electronic Band Structure of Carbon Nanotubes:-}

In order to examine the electronic structure of carbon nanotubes, it is necessary to define the structural configuration. Carbon nanotube structures are defined by the indices $(n, m)$ inscribed in the chiral vector. The chiral vector is a vector along the perimeter of the carbon nanotube.

\section{Crystal lattice:-}

The primitive cell of a carbon nanotube can be described from the unit vectors:

$\vec{a}_{1}=\frac{\sqrt{3}}{2} a \hat{x}+\frac{1}{2} a \hat{y}$

$\vec{a}_{2}=\frac{\sqrt{3}}{2} a \hat{x}-\frac{1}{2} a \hat{y}$

Where $\vec{a}_{1}$ and $\vec{a}_{2}$ are the unit cell vectors, $a$ is the lattice constant of graphene with the value $a=\sqrt{3} a_{c-c}=2.46 \AA$, Where $a_{c-c}$ is the nearest distance between two carbon atoms.

\section{Reciprocal lattice:-}

The reciprocal lattice vectors $\vec{b}_{1}$ and $\vec{b}_{2}$ are of the form:

$\vec{b}_{1}=\frac{2 \pi}{\sqrt{3} a} \hat{x}+\frac{2 \pi}{a} \hat{y}$

$\vec{b}_{2}=\frac{2 \pi}{\sqrt{3} a} \hat{x}-\frac{2 \pi}{a} \hat{y}$

\section{Energy dispersion relation:-}

The energy dispersion relation for carbon nanotubes can be calculated from the electronic structure of graphene. The energy dispersion of graphene is given by (Heo, 2008):

$E(k)= \pm \gamma \sqrt{1+4 \cos \left(\frac{\sqrt{3} a k_{y}}{2}\right) \cos \left(\frac{a k_{x}}{2}\right)+4 \cos ^{2}\left(\frac{a k_{x}}{2}\right)}$

Where $\gamma$ is the transfer integral and $k$ is the wave vector, $k_{x}$ and $k_{y}$ are denote the allowed reciprocal wave vectors along the tube and the circumference axis are found using the relation:

$k_{x}=\frac{(2 n+m) b_{1}+(2 m+n) b_{2}}{N d_{R}}$

$k_{y}=\frac{m b_{1}-n b_{2}}{N}$

Where $N$ is the number of hexagons within a unit cell and it is given by:

$N=\frac{2\left(n^{2}+m^{2}+n m\right)}{d_{R}}$

where $d_{R}$ the greatest common divisor of $(2 n+m)$ and $(2 m+n)$.

One-dimensional (1D) energy band structure of carbon nanotubes can be adopted from Eq. (2.4.5). For armchair and zigzag carbon nanotubes the energy band structures as follows:

$E_{1 D, a r m}(k)= \pm \gamma \sqrt{1+4 \cos \left(\frac{q \pi}{n}\right) \cos \left(\frac{k a}{2}\right)+4 \cos ^{2}\left(\frac{k a}{2}\right)}$

where $k a$ is $-\pi \leq k a \leq \pi$ and $\mathrm{q}$ is $0 \leq q \leq 2 n-1$,

$E_{1 D, z i g}(k)= \pm \gamma \sqrt{1+4 \cos \left(\frac{\sqrt{3} \mathrm{k} a}{2}\right) \cos \left(\frac{q \pi}{n}\right)+4 \cos ^{2}\left(\frac{q \pi}{n}\right)}$

where $k a$ is $-\frac{\pi}{\sqrt{3}} \leq k a \leq \frac{\pi}{\sqrt{3}}$ and $q$ is $0 \leq q \leq 2 n-1$

where $\gamma$ is the transfer integral and \pm corresponds to the valance and the conduction band of the nanotubes. 


\section{Energy gap of carbon nanotubes:-}

Furthermore, the $(n, m)$ integers also determine whether a CNT is metallic or semiconducting. When $n-m$ is a multiple of 3 , the nanotube is metallic, otherwise the nanotube is semiconducting with an energy gap depending on its diameter as follows:

$E_{g, v}=\sqrt{3} a \gamma \frac{2 \pi}{n a}\left(v-\frac{2 n}{3}\right)=\sqrt{3} a \gamma \frac{2}{d}\left(v-\frac{2 n}{3}\right)$

where $d=\frac{n a}{\pi}$ is tube diameter and $v=1, \ldots \ldots 2 n$

$E_{g}=\frac{2 \gamma a_{c-c}}{d}=\frac{0.72 \mathrm{eV}}{d(\mathrm{~nm})}$

where $a_{c-c}$ is the nearest distance between two carbon atoms which is equal to $0.144 \mathrm{~nm}$ and $d$ is the diameter carbon nanotubes. The density of states of carbon nanotubes are given by the following formula:

$g(E)=\sum_{v}^{a l l ~ b a n d} \frac{8}{3 \pi a_{c-c} \gamma} \frac{|E|}{\sqrt{E^{2}-E_{g, v}^{2}}}$

This equation is valid for all semiconductor and metallic carbon nanotubes.

\section{Discussion:-}

From section 2.4. we have seen how the energy band structure depends on the wave vector of a given nanotubes. In order to identify whether the given nanotube is metallic or semiconducting, one can simulate the wave vector dependence of energy band structure of carbon nanotubes. With the help of equation (2.4.9) for armchair tubes and equation (2.4.10) for zigzag tubes, we simulate the variation of energy band structure of the tubes as a function of wave vector as showed in figure 7 respectively using MATLAB codes.

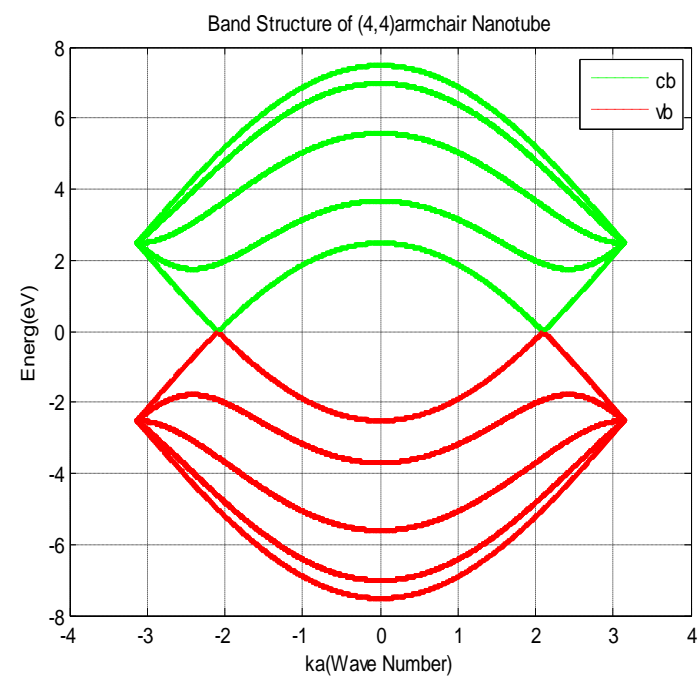

a) $(4,4)$ armchair tube

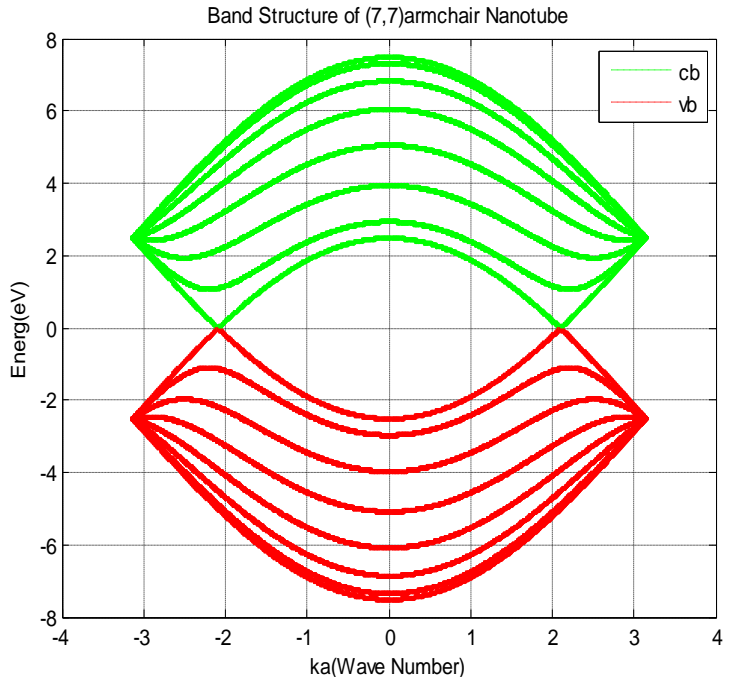

b) $(7,7)$ armchair tube

Figure 7:- Band structure of armchair tubes as function of wave vector using Eq. 2.4.9

From figure $7 \mathrm{a}$ we observe that for $(4,4)$ armchair tube has 10 different subbands; half of the total belongs to conduction and half of the total belongs to valence bands. From the half of conduction and/or valance bands two of them are non-degenerate and the remaining three subbands are degenerate. We also observe that the first conduction and the first valance bands meet at a Fermi level $(k a= \pm 2.032)$. Because of the degeneracy point between the valance and conduction bands at the band crossing, the $(4,4)$ nanotube is thus a zero-gap semiconductor. This implies that the tube is metallic. This discussion is also true for $(7,7)$ armchair tubes as shown in figure $7 \mathrm{~b}$ except the number of subbands and the degenerate. In general, all $(\mathrm{n}, \mathrm{n})$ armchair nanotubes yield $2 \mathrm{n}$ conduction subbands and $2 \mathrm{n}$ valence bands, and of these bands, two are non-degenerate and $n-1$ are doubly degenerate. They all have a band degeneracy between the highest valence band and the lowest conduction band at $(k a= \pm 2.032)$ where the bands cross the Fermi level. The degeneracy comes from the two subbands with the same energy dispersion, but different $q$ values. All armchair nanotubes are expected to exhibit metallic conduction. 


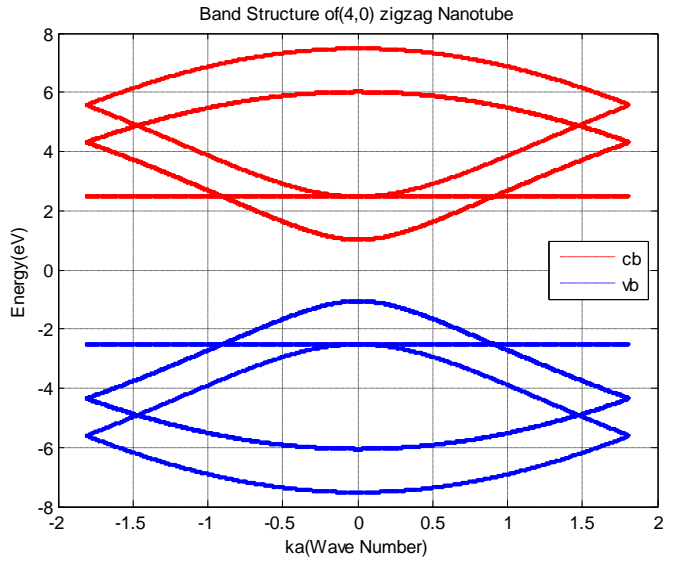

a) $(4,0)$ zigzag tube

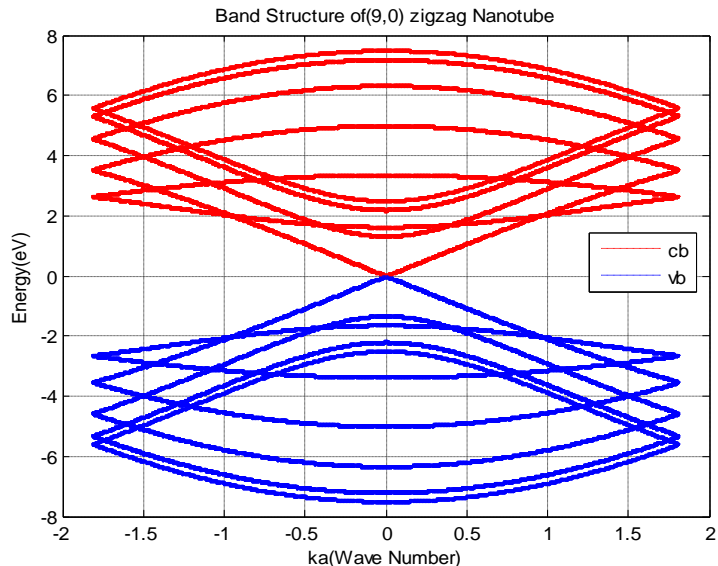

b) $(9,0)$ zigzag tube

Figure 8:- Band structure of zigzag tubes as function of wave vector using Eq. 2.4.10

From figure $8 \mathrm{a}$ we observe that there are 10 sub bands in $(4,0)$ tubes. Half of the total belongs to the conduction band (red color) and half of the total belongs to the valence band (blue color). We observe that the valence and the conduction bands are symmetric with respect to Fermi energy $(E=0)$. Again from figure 8 a the valance bands and the conduction bands do not meet along the wave vector ka. Therefore, this tube is a semiconducting one since there is a band gap. In general most of the zigzag nanotubes are $(n, 0)$ a semiconducting. But when $\mathrm{n}$ is a multiple of 3 , there is no energy gap, then the zigzag nanotubes are metallic, otherwise semiconducting. An example of metallic zigzag nanotube is $(9,0)$. Because as we observed from figure $8 \mathrm{~b}$ above, the first valence and conduction band meet at $k a=0$. Simply there is no energy gap between the conduction and valence band in $(9,0)$ zigzag tubes.

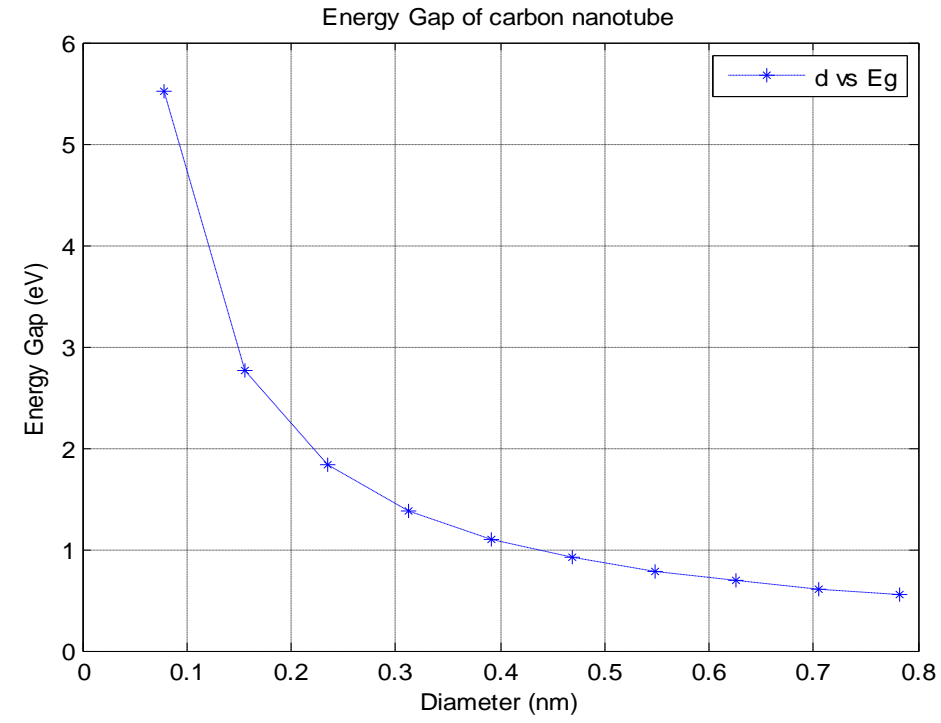

Figure 9:- Energy gap as a function of tube diameter using Eq. 2.4.11

From figure 9 we observe that the band gap for semiconducting tubes is found to be inversely proportional to the tube diameter. The energy gap decreases for larger tube diameters and increases for smaller diameters. Most of the time SWCTs have high energy gap when we compared to MWCTs. The diameter of MWCTs is greater than that of SWCTs. It has been shown that electronic conduction mostly occurs through the external tube for MWNTs. 


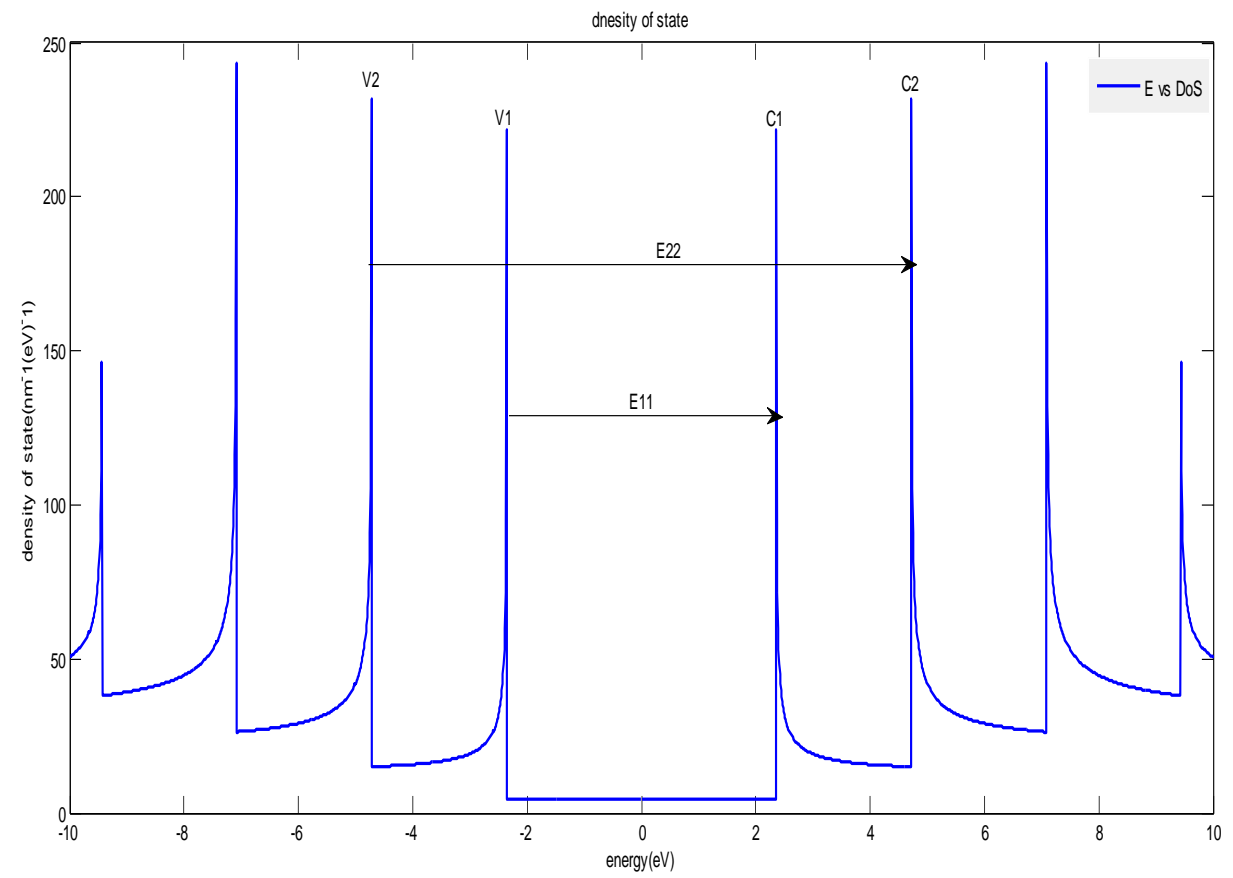

a) Density of state for metallic tube

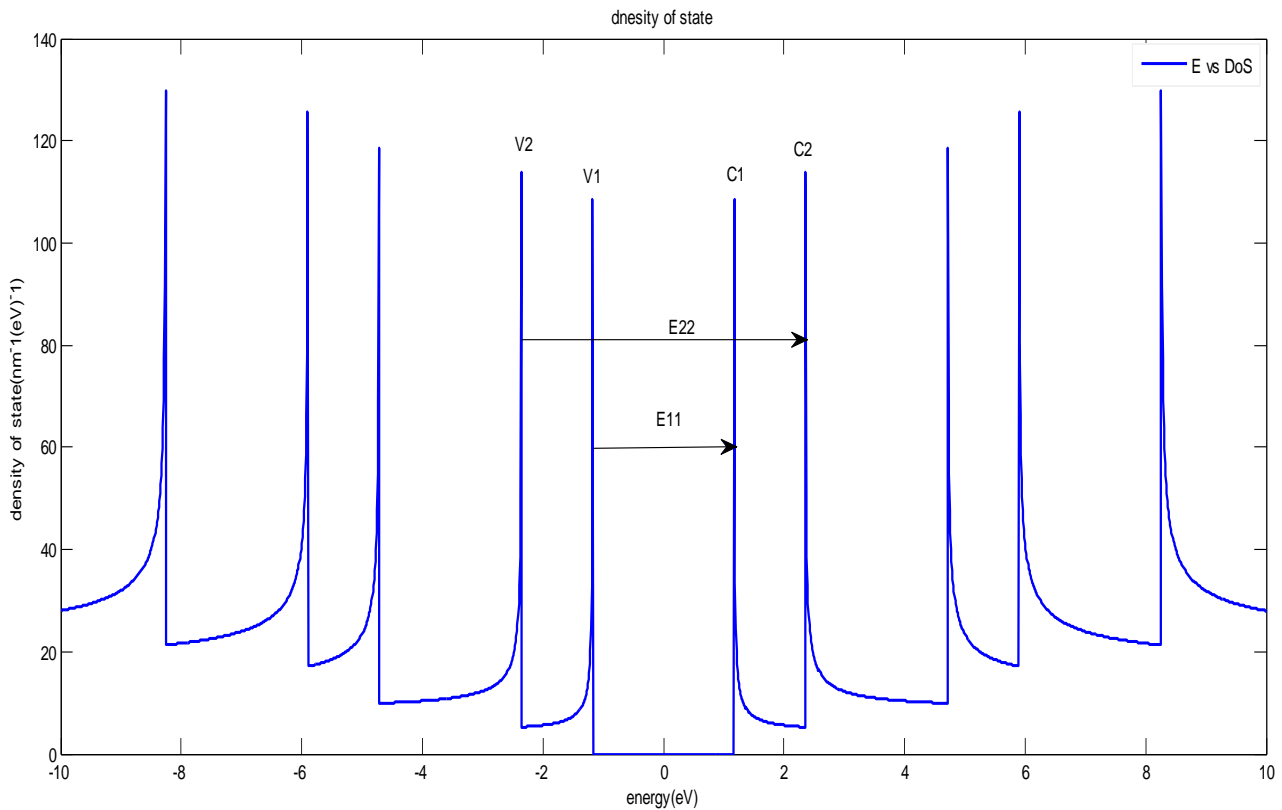

b) Density of state of semiconductor carbon nanotube

Figure 10:- Density of state as function of energy band structure of the carbon nanotubes using Eq. 2.4.13

From figure 10a we observe that at the Fermi energy (the highest occupied energy level) the density of states finite for a metallic tubes (though very small) and from figure $10 \mathrm{~b}$ we observe that at the Fermi energy the density of state is zero for semiconducting tubes. As the energy increased, the sharp peaks in the density of states called the van Hove singularities, appear and specific energy levels. The density of state is highest at the energies where the band structure has a smallest slope and is lowest at the energies where the band structure is steepest. As we observe from the figures the density of state is not continuous function of energy but it descends gradually and then increases in a discontinuous spike. It is clear that the density of state is inversely proportional to the square root of energy in both 
tubes, (i.e., semiconducting and metallic tubes). Van Hove singularities results a remarkable optical properties of carbon nanotubes:

The energies between the van Hove singularities depend on the nanotube structure. Thus by varying this structure, one can tune the optoelectronic properties of carbon nanotube. Such fine tuning has been experimentally demonstrated using UV illumination of polymer-dispersed CNTs (Iakoubovskii et al., 2006).

Optical transitions occur between the $V_{1} \rightarrow C_{1}, V_{2} \rightarrow C_{2}$ and so on states of semiconducting or metallic nanotubes and are traditionally labeled as $S_{22}, S_{22}, M_{11}$, and so on or, if the conductivity of the tube is unknown or unimportant, as $E_{11}, E_{22}$ and so on. Crossover transitions $V_{2} \rightarrow C_{1}, V_{1} \rightarrow C_{2}$ and so on are dipole-forbidden and thus are extremely weak, but they were possibly observed using cross-polarized optical geometry (Miyauchi et al., 2006).

\section{Conclusion:-}

In this project work synthesis, properties and technological applications of carbon nanotubes are reviewed. From the reviewed the synthesis methods chemical vapor deposition method is the most effective method which produces large quantities and high quality of carbon nanotubes. The technique is commonly used for the industrial purposes because the method is already well investigated and offers acceptable results on the industrial-scale.

The electronic band structure of a semiconductor consists of a valence band, conduction band, and Fermi level that are at energy levels particular to a given semiconductor. The Fermi level is the energy of the highest occupied state of electrons at absolute zero, and the valence band contains the valence electrons that when excited can move to the higher energy levels of the conduction band.

The electronic band structure calculations predict that the $(n, m)$ indices determine the metallic or semiconducting behavior of CNT. It is clear that the result shows zigzag tubes $(n, 0)$ should have two distinct types of behavior; metallic when $\mathrm{n} / 3$ is an integer, and otherwise semiconductors. The gaps of the semiconducting $(n, 0)$ zigzag tubes should depend inversely on diameter and $(n, n)$ armchair tubes band gap is remain independent of diameter.

The energy dependence of the nanotube density of states as where, the density of states for metallic and semiconductor zigzag nanotubes was compared. It was observed and demonstrated that the density of states near the Fermi level located at $E=0$ are zero in pure semiconducting nanotubes. But density of states has a value of nonzero for metallic nanotubes even at the Fermi level located at $E=0$.

\section{References:-}

1. Arepalli, S., Nikolaev, P., Gorelik, O., Hadjiev, V., Holmes, W., Files, B., and Yowell L., 2004. Organic semiconductor-carbon nanotube bundle bilayer field effect transistors with enhanced mobilities and high on/off ratios. Carbon 42. p.1783.

2. Bachilo, S.M., Strano, M.S., Kittrel, C., Hauge, R.H., Smalley, R.E. and Weisman, R.B., 2002. Structureassigned optical spectra of single-walled carbon nanotubes. Science 298. pp. 2361-2366.

3. Belin, T. and Epron, F., 2005. Characterization methods of carbon nanotube. Materials Science and Engineering B 119. pp.105-118.

4. Cantalini, C., Valentini, L., Lozzi, L., Armentano, I., Kenny, J.M. and Santucci, S., 2003. $\mathrm{NO}_{2}$ gas sensitivity of carbon nanotubes obtained by plasma enhanced chemical vapor deposition. Sens. Actuators B 93. pp. 333-337.

5. Chang, H., Lee, J.D., Lee, S.M. and Lee, Y.H., 2001. Adsorption of $\mathrm{NH}_{3}$ and $\mathrm{NO}_{2}$ molecules on carbon nanotubes. Appl. Phys. Lett. 79, pp.3863-3865.

6. Charlier, A., McRae, E., Heyd, R. and Charlier, M.F., 2001. Metal semi-conductor transitions under uniaxial stress for single and double-walled carbon nanotubes. J. Phys. Chem. Solids 62, pp.439-444.

7. Dresselhaus, M.S., Dresselhaus, G., Charlier, J.C. and Hern'andez, E., 2004. Electronic, thermal and mechanical properties of carbon nanotubes.

8. Fakhrabadi, M.M., Allahverdizadeh, A., Norouzifard, V. and Dadashzadeh, B., 2012. Mechanical characterization of deformed carbonnanotubes. Digest Journal of Nanomaterials and Biostructures Vol. 7, No. 2, pp.717- 727.

9. Fantini, C., Jorio, A., Souza, M., Strano, M.S., Dresselhaus, M.S. and Pimenta, M.A., 2004. Optical Transition Energies for Carbon Nanotubes from Resonant Raman Spectroscopy, Environment and Temperature Effects. Physical Review Letters 93, No. 14, p 147406. 
10. Fathi, D., 2009. Novel approach for stability analysis in carbon nanotubes interconnects. Journal and Magazine vol. 30, pp.473-477.

11. Guo, C.X., Yang, H.B., Sheng, Z.M., Lu, Z.S., Song, Q.L. and Li, C.M., 2010. Layered Graphene/Quantum Dots for Photovoltaic Devices. AngewandteChemie. Vol. 122, Issue 17, pp.3078 - 3081.

12. Guo, T., Nikolaev, P., Thess, A., Colbert, D.T. and Smalley, R.E., 1995. Large-scale production of single walled carbon nanotubes by the electric-arc technique. Chem.Phys Lett. 243, pp. 49-54.

13. Gupta, N., Alapatt, G.F., Podila, R., Singh, R., and Poole, K.F., 2009. Prospects of Nanostructure Based Solar Cells for Manufacturing Future Generations of Photovoltaic Modules. International Journal of Photoenergy, Article ID 154059, p.13.

14. Hanna, M.C., Ellingson, R.J., Beard, M., Yu, P., Micic, O.I. and Nozik, A.J., 2004. Quantum Dot Solar Cells: High Efficiency through Multiple Exciton Generation Conference Paper Presented at the DOE Solar Energy Technologies.

15. Hasan, T., Sun, Z. and Ferrari, A.C., 2009. Nanotube-polymer composites for ultrafast photonics. Advance Materials, Vol. 21, No. 38-39, pp. 3874-3899.

16. Heo, J., 2008. Probing electronic properties of carbon nanotubes .Thesis In Partial Fulfillment of the Requirements for the degree of Doctor of Philosophy. California institute of technology, Pasadena, California

17. http://en.wikipedia.org/wiki/ Carbonnanotube. Accessed on December 28, 2012.

18. http://en.wikipedia.org/wiki/Chemical vapor deposition. Accessed on December 26, 2012.

19. http://students.chem.tue.nl/ifp03/synthesis.html.Accessed on December 26, 2012.

20. Iakoubovskii, K., Nobutsugu, M., Yeji,K., Kanae, M., Said, K. and Balakrishnan, N., 2006. Midgap Luminescence Centers in Single-Wall Carbon Nanotubes Created by Ultraviolet Illumination. Applied Physics Lett. 89 No.17, p. 173108.

21. Isaacs, J.A., Tanwani, A., Healy, M.L. and Dahlben, L.J., 2010. Economic assessment of single-walled carbon nanotube processes. J. Nanopart Res, 12, pp.551-562.

22. Javey, A., Kim, H., Brink, M., Wang, Q., Ural, A., Guo,J., Mcientyre, P., McEuen, P., Lundstrom, M. and Dai, H., 2002. High k-dielectric for advanced carbon nanotube transistors and logic gates. Nature Materials, vol. 1, pp.241-246.

23. Jin Kim S., Won, J.K., Yudhisthira, S., Alexander, N.C. and Paras, N.P., 2008. Multiple exciton generation and electrical extraction from a PbSe quantum dot photoconductor. Appl. Phys. Lett. 92, 031107.

24. Kataura, H., Kumazawa, Y., Maniwa,Y. and Umezu, I., 1999. Optical Properties of Single-Wall Carbon Nanotubes. Synthetic Metals.vol. 103, pp.2555-2558.

25. Kong, J., Franklin,N.R., Zhou, C., Chapline, M.G., Peng, S., Cho, K. and Dai H., 2000. Nanotube molecular wire as chemical sensors. Science. vol. 287, pp.622-625.

26. Kongkanand, R., Domínguez, R.M. and Kama, P.V., 2007. Single Wall Carbon Nanotube Scaffolds for Photoelectrochemical Solar Cells: Capture and Transport of Photogenerated Electrons. Nano Lett. 7, No. 3, pp.676-680.

27. Kymakis, E., Alexandrou, I. and Amaratunga, A.J., 2003. High open circuit voltage photovoltaic devices from carbon nanotube polymer composites. Progress in Photovoltaics:Research and Applications. 93, No. 3, pp.1764-1768.

28. Lee, J.U., 2005. Photovoltaic effect in ideal carbon nanotube diodes. Appl. Phys.Lett. 87, 073101.

29. Li, J., Lu, Y., Ye, Q., Cinke, M., Han, J. and Meyyappan, M., 2003. Carbon nanotubes sensors for gas and organic vapor detection, Nano Lett.3, pp. 929-933.

30. Lidorikis, E. and Ferrari A. C., 2009. Photonics with multiwall carbon nanotube arrays. ACS NanoLett.3, pp.1238-1248.

31. Loh, K.P., Bao, Q.L., Eda, G. and Chhowalla, M., 2010. Graphene oxide as a chemically tunable platform for optical applications. Nature Chemistry, Vol. 2, No. 12, pp. 1015-1024.

32. Maruyama, B. and Alam, K., 2002. Carbon nanotubes and nanofibers in composite materials. Sampe Journal.38, pp.59-70.

33. Miyauchi,Y., Oba, M. and Maruyama, S., 2006. Cross-Polarized Optical Absorption of Single-Walled Nanotubes Probed by Polarized Photoluminescence Excitation Spectroscopy. Physical Review B 74, No.20, p.205440.

34. Prasada, S.,Singh, M., Shinjini S. and Gambhir, I.S., 2008. Nanotechnology in medicine and antibacterial effect of silver nanoparticles. Digest Journal of Nanomaterials and Biostructures. Vol. 3, No.3, pp. 115 - 122.

35. Puech, P., Hubel, H., Dunstan, D., Bacsa, R. R., Laurent, C. and Bacsa, W.S., 2004. Discontinuous tangential stress in double wall carbon nanotubes. Phys. Rev. Lett. 93, 095506. 
36. Ruan, X., Hua, B. and Timothy S. F., 2010. Optical properties of ordered vertical arrays of multi walled carbon nanotubes from FDTD simulations. School of Mechanical Engineering, Birck Nanotechnology Center, and Energy Center Purdue University.

37. Saito, R., Dresselhaus, G. and Dresselhaus, M.S., 2000.Triogonal Warping Effect of Carbon Nanotubes. Phys. Rev. B 61, pp.2981-2990.

38. Singh, R., Gupta, N. and Poole, K.F., 2008. Global energy conversion revolution in the $21^{\text {st }}$ century through solid state devices in Proceedings of the $26^{\text {th }}$ International Conference on Microelectronics (ICM 08). vol. 1, pp. $45-54$.

39. Stubbs1, S.K., Hardman, S. J.O., Graham, D.M., Spencer, B.F., Flavell, W.R., Glarvey, P., Masala. O., Pickett, N.L. and Binks, D.J., 2010. Efficient carrier multiplication in nanoparticles. Phys. Rev. B 81, 081303.

40. Tripathi, S.M.,Tiwari, S.B. and Shantkriti, S., 2010. Synthesis and Study of Applications of Metal coated Carbon Nanotubes. International Journal of Control and Automation, Vol. 3, No. 2, p. 53.

41. Varghese, O.K., Kichambre, P.D., Gong, D., Ong, K.G., Dickey, E.C. and Grimes, C.A., 2001. Gas sensing characteristics of multi-wall carbon nanotubes. Sens. Actuators, B 81, pp.32-41.

42. Zhang, L. Z., Sun, W. and Cheng, P., 2003. Spectroscopic and Theoretical Studies of Quantum and Electronic Confinement Effects in Nanostructured Materials. MoleculesISSN 1420-3049; pp.207-222. 